\section{Fragile Evidenz: Datenprobleme in der Risikobewertung für Chemikalien}

\author{
von Martin Scheringer, ETH Zürich
}

Für die Risikobewertung von Chemikalien werden Daten zu den physikalischen, chemischen und biologischen Eigenschaften aller kommerziell relevanten Chemikalien benötigt. Die Anzahl der Substanzen, deren Daten bislang erhoben worden sind, liegt jedoch unter fünf Prozent der insgesamt ca. 100.000 Industriechemikalien. Die Chemikalienverordnung der EU von 2007, REACH, verlangt im Prinzip, dass die chemische Industrie die fehlenden Daten erhebt. Erste Untersuchungen der nun unter REACH eingehenden Daten zeigen jedoch, dass unter den Daten stark fehlerbehaftete Werte auftreten, und es ist nicht klar, welche und wie viele Daten korrekt oder aber fehlerbehaftet sind. Die bisher verfügbare Evidenz zu den Eigenschaften kommerziell relevanter Chemikalien muss somit als fragil bezeichnet werden.

\section{Historische Entwicklung der Risikobewertung für Chemikalien}

Die Risikobewertung für Chemikalien ist von großer ökonomischer und gesellschaftlicher Bedeutung, da sie die Grundlage für die Marktzulassung von insgesamt mehreren 10.000 chemischen Substanzen bildet. Sie stützt sich auf Daten zu den physikalischen, chemischen und biologischen Eigenschaften der chemischen Substanzen. Ziel der Risikobewertung ist es, mögliche Schäden für Mensch und Umwelt, die durch die Anwesenheit von Chemikalien ausgelöst werden können, abzuschätzen. Für die Zuverlässigkeit dieser Abschätzungen ist somit erstens das Vorhandensein und zweitens die Qualität dieser Stoffdaten essentiell. Erstaunlicherweise sind jedoch beide Aspekte, Verfügbarkeit und Qualität, in vielen Fällen nicht gewährleistet.

Dieses Problem der nicht vorhandenen Evidenz zu essentiellen Eigenschaften vieler ökonomisch relevanter chemischer Substanzen hat in den letzten 15 Jahren zu einem Umdenken geführt und einen tiefgreifenden Umbruch in der
Risikobewertung für Chemikalien ausgelöst. Um diesen Umbruch einordnen zu können, ist es sinnvoll, sich die historische Entwicklung der Risikobewertung für Chemikalien vor Augen zu führen. Dabei liegt der Schwerpunkt auf der Situation in der EU und auf der größten Gruppe von chemischen Produkten, den sog. Industriechemikalien. ${ }^{1}$

Grundlage der Risikobewertung für Chemikalien in der EU ist die Richtlinie 67/546/EWG (EU 1967), welche die Einstufung, Kennzeichnung und Verpackung gefährlicher Stoffe regelt. Diese Richtlinie wurde seit 1967 mehrfach geändert; wichtig ist in diesem Zusammenhang die Richtlinie 79/831/EWG (EU 1979). In dieser Richtlinie wurde eine Unterscheidung von großer Bedeutung eingeführt, nämlich die Unterscheidung von Altstoffen und Neustoffen. Als Altstoffe wurden alle Substanzen bezeichnet, die in der EU vor dem 18.9.1981 auf dem Markt waren; sie wurden in einem eigenen Verzeichnis aufgelistet (EINECS, European Inventory of Existing Commercial Chemical Substances), welches 100.204 Substanzen umfasst. Neustoffe waren dementsprechend Substanzen, die nach dem 18.9.1981 auf den Markt gebracht wurden. Für Neustoffe mussten Hersteller und Importeure die physikalisch-chemischen Eigenschaften der Substanzen sowie deren Toxizität und Ökotoxizität selbst bestimmen. Insgesamt wurden von 1981 bis 2007 ca. 5.500 Neustoffe auf den Markt gebracht. Anders als bei den Neustoffen lag die Verantwortung für die Testung von Altstoffen bei den Behörden. Aufgrund der großen Zahl der Altstoffe wurde für die Risikobewertung eine Prioritätenliste definiert. Dennoch war die Zahl der Altstoffe, für welche bis 1997 eine durch die Behörden der EU durchgeführte Risikobewertung vorlag, beunruhigend klein, sie betrug nämlich nur zehn (EEA 1998).

Dass die Altstoffbewertung auch mit Unterstützung durch das Altstoffprogramm der OECD (OECD 2013) nicht praktikabel war, führte in den 1990er Jahren zur Entwicklung eines neuen Konzepts für den rechtlichen Rahmen der Stoffbewertung in der EU. 2001 wurde das Weißbuch der EU-Kommission veröffentlicht, welches dieses neue Konzept auf knapp 30 Seiten vorstellt (EC 2001). Das neue Konzept wurde als REACH - Registration, Evaluation, Authorisation and Restriction of Chemicals - bezeichnet. Zentrale 
Neuerungen unter REACH sollten gemäß dem Weißbuch u. a. werden:

- Die Unterscheidung zwischen Alt- und Neustoffen wird aufgehoben. Alle Stoffe, die auf dem Markt bleiben sollen, müssen registriert werden.

- Die chemische Industrie ist dafür verantwortlich, die für die Registrierung erforderlichen Stoffdaten $\mathrm{zu}$ erheben und Testkonzepte $\mathrm{zu}$ erarbeiten. Wenn diese Stoffdaten nicht erhoben und eingereicht werden, verschwindet ein Stoff vom Markt (,no data, no market").

- Der Umfang der Daten, die für die Registrierung erhoben werden müssen, nimmt mit dem Produktionsvolumen zu.

- Stoffe mit besonders problematischen Eigenschaften müssen eigens zugelassen werden, sofern sie überhaupt noch verwendet werden sollen. Als besonders problematische Eigenschaften gelten u. a. CMR-Eigenschaften (Kanzerogenität, Mutagenität, Reproduktionstoxizität) und PBT-Eigenschaften (Persistenz, Bioakkumulationspotenzial, Toxizität).

Auf dieser Grundlage wurde die REACH-Verordnung (EU 2006) erarbeitet, die bis heute als die komplexeste einzelne Gesetzgebung der EU gilt. Die REACH-Verordnung ist am 1.6.2007 in Kraft getreten. ${ }^{2}$ Im Kontext des hier behandelten Themas „Fragile Evidenz“ ist zentral, dass ein Hauptziel der REACH-Verordnung darin besteht, ein Regime, das sich durch „Fehlen von Evidenz" charakterisieren lässt, zu beenden.

\section{Illustration des Regimes „Fehlen von Evidenz"}

Das Problem der fehlenden Evidenz lässt sich am Beispiel der PBT-Bewertung illustrieren. Die drei Eigenschaften Persistenz, Bioakkumulationspotenzial und Toxizität fassen zusammen, wodurch eine Substanz zum Umweltproblem werden kann: Persistenz, also Langlebigkeit wegen langsamen chemischen und biologischen Abbaus in der Umwelt, führt dazu, dass die Substanz sich weiträumig verteilen kann und für die Aufnahme durch Organismen lange Zeit zur Verfügung steht. Das Bioakkumulationspotenzial drückt aus, ob die Substanz eine Tendenz hat, sich im Gewebe von Organismen anzureichern, d. h. im Organismus höhere Konzentrationen aufzubauen als in der Umgebung. Die Toxizität schließlich beschreibt, welche Konzentrationen der Substanz toxische Wirkungen in Organismen auslösen. Substanzen, die alle drei Eigenschaften besitzen, werden als PBT-Chemikalien bezeichnet und gelten als besonders umweltgefährdend.

Konkret werden die PBT-Eigenschaften einer chemischen Substanz in folgender Weise bestimmt: Die Persistenz wird anhand von Halbwertszeiten für den Abbau in der Umwelt quantifiziert; diese Halbwertszeiten müssen in Abbautests gemessen werden. In allen rechtlichen Bestimmungen, die eine PBT-Bewertung vorsehen, sind Grenzwerte für die Halbwertszeit festgelegt, z. B. 120 Tage im Boden. Wenn die Halbwertszeit einer Substanz diesen Grenzwert überschreitet, gilt die Substanz als ,persistent“. Das Bioakkumulationspotenzial wird in den meisten Fällen in Form des Biokonzentrationsfaktors (BCF) bestimmt, welcher in Experimenten mit Fischen gemessen wird. In diesen Experimenten wird die Anreicherung zwischen Wasser und dem Gewebe des Fisches bestimmt. Der BCF ist dann das Verhältnis der Konzentration im Gewebe und der Konzentration im Wasser. Eine Substanz gilt als bioakkumulierend, wenn ihr BCF den Wert 2.000 überschreitet. Die Toxizität schließlich wird im PBT-Kontext in Experimenten mit Fischen, Flusskrebsen (Daphnien) und Algen gemessen; Säugetiere werden im PBT-Zusammenhang nicht getestet. Bestimmt wird die Konzentration, bei der 10 Prozent oder 50 Prozent der Organismen im Testsystem Effekte zeigen (Immobilisierung, Wachstumsminderung oder auch Tod). Eine Substanz gilt im PBT-Kontext als toxisch, wenn ihre Effektschwelle für akute Effekte unter $0.1 \mathrm{mg} / 1$ liegt $(0.01 \mathrm{mg} / \mathrm{l}$ bei chronischen Effekten).

Um eine umfassende PBT-Bewertung für eine große Zahl chemischer Substanzen durchzuführen, haben Strempel et al. (2012) für 95.000 Substanzen in öffentlich zugänglichen Datenbanken nach gemessenen Daten für Abbau-Halbwertszeit, BCF und Toxizität gesucht. Um die große Anzahl von Substanzen systematisch behandeln zu können, benutzten sie Methoden zur automatischen Extraktion von Stoffdaten aus den Datenbanken. Gemessene Halbwertszeiten, BCF-Werte und Toxizitäten waren für weniger als 3 Prozent (!) der 95.000 
Substanzen vorhanden. Im Einzelnen ließen sich Halbwertszeiten für 220 Substanzen, BCF-Werte für 995 Substanzen und Toxizitäten für 2.200 Substanzen finden. Bei 2.606 Substanzen (2,7 Prozent) war ein Messwert für mindestens eine der drei Eigenschaften vorhanden, aber nur bei 91 Substanzen $(0,1$ Prozent) waren Messwerte für alle drei Eigenschaften vorhanden (Strempel et al. 2012). Dies ist eine sehr deutliche Illustration des Regimes der fehlenden Evidenz (UNEP 2013).

Um dennoch eine PBT-Bewertung der 95.000 Substanzen durchführen zu können, mussten Strempel et al. (2012) für die große Mehrzahl der Substanzen die benötigten Stoffeigenschaften aus der chemischen Struktur abschätzen. Abschätzmethoden dieser Art sind verfügbar, aber die Resultate sind mit z. T. erheblichen Unsicherheiten behaftet und können nur als sog. „Screening“ (Voruntersuchung) gelten. Unter den 95.000 von Strempel et al. (2012) untersuchten Substanzen befinden sich 2.930 Substanzen (3,1 Prozent), deren (abgeschätzte) PBT-Eigenschaften die REACH-Grenzwerte für PBT-Substanzen überschreiten. Dies sind deutlich mehr als die bisher als mögliche PBT-Substanzen in der EU diskutierten gut 100 Chemikalien (Pavan/Worth 2008, Tab. 5). Insofern ist es sehr sinnvoll, dass unter REACH eine PBT-Bewertung systematisch durchgeführt werden muss.

\section{Datenprobleme unter REACH}

Mit dem Inkrafttreten von REACH wurde auch die Europäische Chemikalienagentur, ECHA, gegründet. Die ECHA ist für die Umsetzung von REACH verantwortlich. Ein erster Schritt unter REACH war die Vorregistrierung aller Substanzen, die auf dem EU-Markt bleiben sollten. Die Vorregistrierung musste bis zum 1.12.2008 vorgenommen werden; es wurden gut 140.000 Substanzen vorregistriert. Danach begann die eigentliche Registrierung, die in drei Stufen abläuft: Bis zum 1.12.2010 mussten Substanzen mit einem Produktionsvolumen von über $1.000 \mathrm{t} / \mathrm{a}$ registriert werden; für Substanzen mit Produktionsvolumina über 100 t/a und über $10 \mathrm{t} / \mathrm{a}$ sind die Registrierungsdaten der 1.6.2013 und der 1.6.2018. Zurzeit sind 10.200 Substanzen registriert worden. Die ECHA unterhält eine Datenbank, auf welcher ein großer Teil der Stoffdaten aus den Registrierungsdossiers öf- fentlich verfügbar ist (ECHA 2013). Auch dies, die öffentliche Verfügbarkeit von Stoffdaten, ist ein zentrales Ziel der REACH-Verordnung.

An dieser Stelle lässt sich nun fragen, inwieweit die Generierung von Stoffdaten unter REACH die Situation des eklatanten Datenmangels verbessert hat. Einerseits ist festzuhalten, dass die Verfügbarkeit eines ganzen Spektrums von Stoffdaten zu mehr als 10.000 Substanzen ein großer Fortschritt ist. Andererseits zeigen erste Untersuchungen der Daten, dass die Qualität der Daten sehr unterschiedlich ist, und dass eine systematische und umfassende Beurteilung der Qualität der Daten zurzeit kaum möglich ist.

Eine erste Beobachtung ist, dass für viele Substanzen nach wie vor keine Daten in der ECHA-Datenbank vorhanden sind. Stieger et al. (2013) haben für 36 kommerziell relevante bromierte Flammschutzmittel die Verfügbarkeit von PBT-Daten in 25 verschiedenen Datenbanken, u. a. der ECHA-Datenbank, untersucht. In der ECHA-Datenbank waren PBT-relevante Stoffdaten zu lediglich sieben der 36 Substanzen zu finden. Diese Zahl wird jedoch im weiteren Verlauf des Registrierungsprozesses noch zunehmen.

Eine zweite Beobachtung ist, dass es in der ECHA-Datenbank Einträge gibt, die in grober Weise inkorrekt sind. Beispiele sind die OktanolWasser-Verteilungskoeffizienten $\left(K_{\text {ow }}\right)$ sowie die Toxizitätsdaten verschiedener bromierter Flammschutzmittel (Stieger et al. 2013). Der $K_{\text {ow }}$ ist das Verhältnis der Löslichkeit einer Substanz in Oktanol und in Wasser; Oktanol ist dabei ein Ersatz für Lipidsubstanzen wie z. B. Fettgewebe. Der $K_{\text {ow }}$ ist eine gute Grundlage, um den BCF abzuschätzen, und er bildet in Umweltchemie und Ökotoxikologie eines der zentralen Charakteristika von organischen Chemikalien. Der $K_{\text {ow }}$ wird oft in logarithmierter Form als $\log K_{\text {ow }}$ angegeben. Für die Substanz Decabromdiphenylethan findet sich in der ECHA-Datenbank ein $\log K_{\text {ow }}$ von $3.55 .^{3}$ Aus der chemischen Struktur der Substanz und aus den Eigenschaften sehr ähnlicher Substanzen lässt sich jedoch ableiten, dass der $\log K_{\mathrm{ow}}$ von Decabromdiphenylethan bei deutlich über 10 liegen müsste (Stieger et al. 2013). Mit anderen Worten, der $K_{\text {ow }}$, der vom Hersteller der Substanz bei der ECHA eingereicht wurde und nun in ECHA-Datenbank angegeben ist, liegt mit Sicherheit um ei- 
nen Faktor von mehr als einer Million zu tief. Dies ist durchaus relevant, weil die Substanz bei einem so tiefen $\log K_{\text {ow }}$ als wenig toxisch und bioakkumulierend eingeschätzt wird; bei höherem $\log K_{\text {ow }}$ wäre dies nicht der Fall. Umso erstaunlicher ist, dass die ECHA diesem Wert höchste Zuverlässigkeit attestiert (,,reliable without restriction“). Weiterhin ist erstaunlich, dass der falsche Wert nach wie vor in der Datenbank zu finden ist, obwohl die ECHA mehrfach von verschiedener Seite darauf hingewiesen wurde, dass der Wert falsch ist.

Ein weiteres Beispiel ist der Eintrag zum $K_{\text {ow }}$ von Tetrabromphthalsäureanhydrid: Hier findet man in der ECHA-Datenbank die Erläuterung, dass es sich gar nicht um einen Oktanol-WasserVerteilungskoeffizienten handelt, sondern um einen Verteilungskoeffizienten für 1,2-Dichlorbenzol und Wasser. Dieser hat jedoch im Vergleich zum $K_{\text {ow }}$ eine viel geringere Aussagekraft.

In anderen Datenbanken, z. B. dem „High Production Volume Information System" der US Environmental Protection Agency und der Altstoffdatenbank der OECD, finden sich weitere Beispiele für $K_{\mathrm{ow}}$-Werte, die um mehrere Größenordnungen zu tief sind (Stieger et al. 2013). Generell ist bekannt, dass hohe $K_{\text {ow }}$-Werte schwer zu messen sind, weil die Konzentrationen solcher Substanzen in Wasser sehr gering sind, und zu tiefe $K_{\text {ow }}$-Werte lassen sich ohne weiteres als Messfehler erklären. In den Datenbanken gibt es jedoch keine Hinweise dieser Art.

Fehlerhafte Einträge finden sich auch unter den Toxizitätsdaten. Ein bei den 36 bromierten Flammschutzmitteln häufig auftretendes Problem ist, dass Wirkungskonzentrationen angegeben werden, welche zum Teil um mehrere Größenordnungen über der Wasserlöslichkeit der Substanzen liegen, also rein physikalisch gar nicht möglich sind (in der ECHA-Datenbank ist es wiederum Decabromdiphenylethan, wo dieses Problem auftritt). Eine mögliche Ursache für diesen Befund ist, dass nicht die tatsächlich im Wasser gelösten Konzentrationen angeführt werden, sondern nur die nominalen Konzentrationen, wobei ein erheblicher Teil der Substanz z. B. an den Wänden des Gefäßes adsorbiert sein oder als ungelöster Feststoff vorliegen kann. Eine andere mögliche Ursache dieses Befundes ist, dass neben der Testsubstanz auch Hilfsstoffe zum Erhöhen der Löslichkeit eingesetzt wurden, welche ihrerseits auch toxische Effekte haben können. In beiden Fällen sind die angegebenen Resultate ohne jede Aussagekraft, und sie sollten nicht in Stoffdatenbanken aufgeführt werden. Es ist bekannt, dass die Bestimmung der Toxizität von Substanzen mit geringer Wasserlöslichkeit experimentell schwierig ist (Mayer/Reichenberg 2006). Wie bei Mayer/Reichenberg (2006) dargestellt, heißt dies jedoch gerade nicht, dass solche Substanzen nicht toxisch wären.

\section{Schlussfolgerungen}

Die Beobachtungen zu den Stoffdaten der bromierten Flammschutzmittel in den verschiedenen Datenbanken führen zu folgenden allgemeineren Feststellungen zur Handhabung von Chemikaliendaten unter der REACH-Verordnung:

1. Für die Registrierung unter REACH gilt ein enger und rigider Zeitplan ohne jede Flexibilität. Um die für die Registrierung erforderlichen Daten innerhalb der gesetzten Fristen beizubringen, trägt die chemische Industrie u. a. auch bereits vorhandene, z. T. mehrere Jahrzehnte alte Daten zusammen.

2. Zumindest eine Reihe von Eingaben der chemischen Industrie erscheinen unkorrigiert in der ECHA-Datenbank. Dies steht tendenziell im Gegensatz zum eigentlich vorgesehenen Ablauf, nach dem die ECHA mindestens fünf Prozent der eingehenden Registrierungsdossiers inhaltlich prüften soll.

3. Die ECHA-Datenbank ermöglicht es nicht, Stoffdaten mehrerer Substanzen gleichzeitig $\mathrm{zu}$ extrahieren. Für jeden Datenpunkt muss man sich von Hand durch mehrere Seiten hindurchklicken. Dadurch wird es unmöglich, die Daten von hunderten oder tausenden von Substanzen systematisch zu vergleichen. Wie bei Stieger et al. (2013) gezeigt, fallen inkorrekte Werte schnell auf, wenn man z. B. die $K_{\text {ow }}$-Werte für eine Reihe von Substanzen, von denen man weiß, dass ihr $K_{\text {ow }}$ systematisch zunimmt, graphisch darstellt, und die einzelnen $K_{\text {ow }}$-Messwerte mit dieser bekannten Beziehung vergleicht.

4. Die ECHA kann Registrierungen nicht widerrufen oder entziehen, auch wenn die Datenqualität mangelhaft ist (UBA 2012). Somit 
sind Registrierungen möglich, wenn die $\mathrm{Da}-$ tenanforderungen rein formal erfüllt, die eigentlichen Werte aber fehlerhaft sind.

Als Folgerung ergibt sich: Im Rahmen des Registrierungsprozesses unter REACH wird zwar zunehmende Evidenz zu Stoffeigenschaften aufgebaut, doch diese Evidenz ist fragil. Weil die Datenbank mit Sicherheit Stoffdaten enthält, die mit erheblichen Fehlern behaftet sind, und weil gleichzeitig nicht bekannt ist, welche und wie viele Stoffdaten insgesamt fehlerbehaftet sind, ist die Datenbank als Ganze nicht zuverlässig (dies gilt nicht nur für die ECHA-Datenbank, sondern auch für andere Datenbanken für physikalische, chemische und biologische Stoffeigenschaften). Da die ECHA-Datenbank auch keine verlässlichen Warnungen vor fehlerhaften Daten enthält, müssen Parteien, welche mit den Stoffdaten arbeiten möchten (z. B. Umweltorganisationen, Privatpersonen), jeden Eintrag selbst prüfen. Dies erfordert substantielles umweltchemisches und ökotoxikologisches Wissen und bedeutet erheblichen Zusatzaufwand auf Seiten aller Parteien, die die Stoffdaten verwenden möchten.

Die Situation ließe sich durchaus verbessern, wenn die ECHA die eingehenden Daten im Detail prüfen würde. Für verschiedene Stoffeigenschaften stehen Methoden zur Überprüfung der Daten zur Verfügung. Allerdings müssten auf Seiten der ECHA die personellen und finanziellen Ressourcen, die für die Prüfung (die „Evaluierung" im Namen der REACH-Verordnung) zur Verfügung stehen, deutlich erhöht werden. Ein zentrales Ziel der REACH-Verordnung könnte verfehlt werden, wenn die eingehenden Stoffdaten nicht gründlicher geprüft und ggf. auch korrigiert werden, als dies zurzeit der Fall ist.

\section{Anmerkungen}

1) Es ist zweckmäßig, chemische Substanzen nach den rechtlichen Vorgaben für ihre Zulassung einzuteilen. Unterschieden werden u. a. Pflanzenschutzmittel, Biozide, Pharmazeutika sowie Industriechemikalien. Die ersten drei Gruppen umfassen jeweils einige 100 Substanzen (Wirkstoffe). Substanzen, die keiner dieser drei Gruppen angehören, werden unter dem Sammelbegriff „Industriechemikalien“ zusammengefasst. Die Gruppe der Industriechemi- kalien umfasst ca. 100.000 Substanzen. Die Anforderungen an die Risikobewertung (Art und Umfang der Testung) unterscheiden sich für die verschiedenen Gruppen. Da Industriechemikalien nicht darauf ausgelegt sind, biologisch aktiv zu sein, sind die Testanforderungen für sie am geringsten.

2) Einen Überblick über REACH gibt das Umweltbundesamt unter http://www.reach-info.de; eine Diskussion der REACH-Verordnung findet sich z. B. bei Abelkop et al. 2013.

3) Diese und weitere Angaben zu Daten aus der ECHA-Datenbank lassen sich verifizieren, indem man auf der Seite http://echa.europa.eu/informa tion-on-chemicals/registered-substances die Daten der hier erwähnten Substanzen aufsucht.

\section{Literatur}

Abelkop, A.; Botos, A.; Wise, L.R. et al., 2013: Regulating Industrial Chemicals: Lessons for U.S. lawmakers from the European Union's REACH Program. Indiana; http://www.indiana.edu/ spea/faculty/pdf $/ \mathrm{REACH}_{-}$ report.pdf (download 3.10.13)

EC - European Commission, 2001: White Paper. Strategy for a Future Chemicals Policy. Brüssel

ECHA - European Chemicals Agency, 2013: Registrierte Stoffe. http://echa.europa.eu/information-onchemicals/registered-substances (download 3.10.13)

EEA - European Environment Agency, 1998: Chemicals in the European Environment: Low Doses, High Stakes. Copenhagen

EU - Europäische Union, 1967: Richtlinie des Rates vom 27. Juni 1967 zur Angleichung der Rechtsund Verwaltungsvorschriften für die Einstufung, Verpackung und Kennzeichnung gefährlicher Stoffe (67/548/EWG). Brüssel

EU - Europäische Union, 1979: Richtlinie des Rates vom 18. September 1979 zur sechsten Änderung der Richtlinie 67/548/EWG zur Angleichung der Rechtsund Verwaltungsvorschriften für die Einstufung, Verpackung und Kennzeichnung gefährlicher Stoffe (79/831/EWG). Brüssel

EU - Europäische Union, 2006: Verordnung (EG) Nr. 1907/2006 des Europäischen Parlaments und des Rates vom 18. Dezember 2006 zur Registrierung, Bewertung, Zulassung und Beschränkung chemischer Stoffe (REACH), zur Schaffung einer Europäischen Agentur für chemische Stoffe, zur Änderung der Richtlinie 1999/45/EG und zur Aufhebung der Verordnung (EWG) Nr. 793/93 des Rates, der Verordnung (EG) Nr. 1488/94 der Kommission, der Richtlinie 76/769/ EWG des Rates sowie der Richtlinien 91/155/EWG, 
93/67/EWG, 93/105/EG und 2000/21/EG der Kommission. Brüssel

Mayer, P.; Reichenberg, F., 2006: Can Highly Hydrophobic Organic Substances Cause Aquatic Baseline Toxicity and Can They Contribute to Mixture Toxicity? In: Environmental Toxicology \& Chemistry 25 (2006), S. 2639-2644

OECD - Organisation for Economic Co-operation and Development, 2013: OECD Cooperative Chemicals Assessment Programme. Paris; http://www.oecd. org/env/ehs/risk-assessment/oecdcooperativechemicalsassessmentprogramme.htm (download 3.10.13)

Pavan, M.; Worth, A., 2008: A Set of Case Studies to Illustrate the Applicability of DART (Decision Analysis by Ranking Techniques) in the Ranking of Chemicals. Ispra: European Commission Joint Research Centre; http://ihcp.jrc.ec.europa.eu/our_labs/predictive_toxicology/doc/EUR_23481_EN.pdf (download 3.10.13)

Stieger, G.; Scheringer, M.; Ng, C.A. et al., 2013: Assessing the Persistence, Bioaccumulation Potential and Toxicity of BFRs: Data Availability and Data Quality for 36 Alternative BFRs. BFR 2013 Symposium, April 2013, San Francisco, USA. http:// www.bfr2013.com/upload/abstract-download/2013// Poli/141110_BFR2013_Stieger_etal_03\%20(2).pdf (download 3.10.13)

Strempel, S.; Scheringer, M.; Ng, C.A. et al., 2012: Screening for PBT Chemicals among the "Existing" and "New" Chemicals of the EU. In: Environmental Science \& Technology 46 (2012), S. 5680-5687

UBA - Umweltbundesamt, 2012: Informationslage zu Chemikalien verbessert. Fünf Jahre REACH - Positive Bilanz trotz sichtbarer Defizite. Dessau; http:// www.umweltbundesamt.de/sites/default/files/medien/press/pd12-020_informationslage_zu_chemikalien_verbessert.pdf (download 3.10.13)

UNEP - United Nations Environment Programme, 2013: Minimizing Chemical Risks. In: UNEP: Year Book 2013. Nairobi; http://www.unep.org/yearbook/2013/ (download 12.11.13)

\section{Kontakt}

PD Dr. Martin Scheringer

Eidgenössische Technische Hochschule Zürich 8093 Zürich, Schweiz

Tel.: +41 446323062

E-Mail: scheringer@chem.ethz.ch

\section{Nachweis erbracht - Mission erfüllt!?}

\section{Zur Zukunft des Weltklimarats}

\section{von Silke Beck, Helmholtz-Zentrum für Umweltforschung - UFZ, Leipzig}

Dieser Beitrag vertritt die These, dass der Weltklimarat seine ursprüngliche Mission, den wissenschaftlichen Nachweis für den menschgemachten Klimawandel zu liefern, längst hinreichend erfüllt hat. Anhand der Ereignisse rund um die Vertragsstaatenkonferenz in Kopenhagen 2009 (COP 15) wird gezeigt, dass der IPCC-Bericht von 2007, der diesen Nachweis bereits erbracht hat, nicht, wie erhofft, zu einem positiven Wendepunkt und Durchbruch der internationalen Klimapolitik geführt hat. Der Beitrag zeigt, dass und warum das szientistische Verständnis von Politik, das von vielen Protagonisten in der internationalen Klimaforschung aufrechterhalten wird, stattdessen zu einer Engführung der politischen Diskussion führt und sich in einem Mangel an politischen Alternativen und an politischer Unterstützung niederschlägt. Von daher sollte die Veröffentlichung der IPCC-Berichte, die sich über das nächste Jahr erstrecken werden, als Gelegenheit genutzt werden, um die Funktionen, Verfahren und Strukturen des Klimarats grundlegend zu überdenken und entsprechend anzupassen.

\section{$1 \mathrm{Zu}$ den Errungenschaften des Weltklimarats}

Eine der wesentlichen Errungenschaften des Weltklimarats besteht darin, dass es ihm gelungen ist, den eindeutigen wissenschaftlichen Nachweis zu erbringen, dass der Klimawandel bereits eingetreten ist und sich auf menschliche Ursachen zurechnen lässt. Für diese Bemühungen wurde er 2007 - zusammen mit dem ehemaligen amerikanischen Vize-Präsidenten Al Gore - mit dem Friedensnobelpreis ausgezeichnet. Dieser Preis gilt vor allem auch als Anerkennung dafür, dass der Weltklimarat nicht nur Bewusstsein für die Risiken des anthropogenen Klimawandels in Öffentlichkeit und Politik geschaffen, sondern auch die erforderlichen politischen Aktivitäten in Gang gesetzt hat. Die Zusammenfassung des Berichts 